\title{
A Roadmap to International Standards Development for Cognitive Radio Systems and Dynamic Spectrum Access
}

\author{
Jim Hoffmeyer \\ Western Telecom Consultants, Inc. \\ United States
}

\section{Introduction}

This chapter provides a summary of standards activities related to cognitive radio systems (CRS) and dynamic spectrum access (DSA) systems. Because of the vast amount of standardization activity in this arena, this chapter can provide only a high-level snapshot of current CRS/DSA standardization activities and a projection of activities expected in the near future. Sufficient references including URL references are included so that the interested reader can review in as much detail as desired the standards that have and are being developed by numerous international standards development organizations (SDOs). These standards will be evolving in time as will be described later in this chapter in the descriptions of the various SDOs. It is expected that standardization in this arena will continue by these SDOs for at least the next decade.

Section 2 sets the stage by describing the terminology that is used by researchers and practitioners because there are many terms closely related to CRS and DSA. As noted in Section 2, there is not total agreement on definitions between the various standards development organizations. Section 3 describes how standards development for CRS/DSA is but one piece of the puzzle that must be solved in order for CRS/DSA systems to achieve their full potential. In that sense, Section 3 can be viewed as the statement of the problem. In order to solve the standardization portion of the puzzle, numerous international SDOs are working on different pieces of the standardization puzzle. The work of these SDOs is described in Sections 4 through 6. A brief summary and a description of future standards work are both provided in Section 7.

\section{Terminology}

The term "cognitive radio" was first coined by Dr. J. Mitola (Mitola, 2000). Since that time the term "cognitive radio" and the acronym "CR" have been used in countless books, journal articles, and telecommunication standards documents. More recently, the term "cognitive radio system" and the acronym "CRS" frequently have been used. The term "cognitive radio systems" has gained international recognition as a result of its adoption by the International Telecommunication Union Radiocommunication Sector. (ITU-R, 2009). It has also been adopted by the US National Telecommunication and Information Administration. (NTIA, 2011). 


\begin{tabular}{|c|c|c|}
\hline Organization & Focus of CRS and DSA Activities & Section \\
\hline ITU-R & $\begin{array}{l}\text { Develops Radio Regulations which is a treaty-level } \\
\text { international agreement. Develops ITU-R Reports and } \\
\text { Recommendations which support the agreements formally } \\
\text { accepted within the Radio Regulations. }\end{array}$ & $\begin{array}{l}\text { Section } \\
4 .\end{array}$ \\
\hline IEEE & The IEEE Dynamic Spectrum Access Networks Standards & Section \\
\hline DySPAN-SC & $\begin{array}{l}\text { Committee develops basic cognitive radio system/dynamic } \\
\text { spectrum access (CRS/DSA) technology standards with the } \\
\text { focus on the improved use of spectrum in any spectrum } \\
\text { band. }\end{array}$ & 5.1 \\
\hline IEEE 802 & $\begin{array}{l}\text { The IEEE } 802 \text { Standards Committee develops standards for } \\
\text { local and regional networks. This organization has focused } \\
\text { on CRS/DSA for TV white space bands. }\end{array}$ & $\begin{array}{l}\text { Section } \\
5.2\end{array}$ \\
\hline Other & $\begin{array}{l}\text { There are many organizations that either directly or } \\
\text { indirectly develop standards related to CRS/DSA or } \\
\text { standards which incorporate CRS/DSA technology as part } \\
\text { of the overall standard. A partial list of these organizations } \\
\text { includes ETSI, Ecma, IEEE ComSoc Technical Committees, } \\
\text { Wireless Innovation Forum WRAN Alliance and the } \\
\text { Wireless Research Forum. These and other organizations } \\
\text { such as GSMA and NGMN provide input to the standards } \\
\text { development process from the research, operator, and other } \\
\text { communities. Some of these organizations develop } \\
\text { specifications based on the standards published by } \\
\text { accredited Standards Development Organizations. An } \\
\text { example is the WiMax Forum which certifies products } \\
\text { based on the IEEE } 802.16 \text { Standard. }\end{array}$ & $\begin{array}{l}\text { Section } \\
6\end{array}$ \\
\hline
\end{tabular}

Some of the difficulty in the terminology is related to the fact that some of the functionality typically associated with cognitive radios is outside the functionality of what is historically associated with a "radio." As will be described in Section 5.1.1, the Institute of Electronics and Electrical Engineers (IEEE) Standard IEEE 1900.1 TM addresses the terminology issue and discusses basic concepts associated with several technologies related to CRS.

There are many terms that are being used in the literature being used that are closely related to CRS technology including dynamic spectrum access, software defined radio, hardware radio, adaptive radio, policy-based radio, reconfigurable radio, and software-controlled radio. In this chapter we focus on standards for CRS and DSA which have been defined as:

- Cognitive Radio System: “A radio system employing technology that allows the system to obtain knowledge of its operational and geographical environment, established policies and its internal state; to dynamically and autonomously adjust its operational parameters and protocols according to its obtained knowledge in order to achieve predefined objectives; and to learn from the results obtained." (ITU-R, 2009).

- Dynamic Spectrum Access: "The real-time adjustment of spectrum utilization in response to changing circumstances and objectives. 
NOTE-Changing circumstances and objectives include (and are not limited to) energyconservation, changes of the radio's state (operational mode, battery life, location, etc.), interferenceavoidance (either suffered or inflicted), changes in environmental/external constraints (spectrum, propagation, operational policies, etc.), spectrum-usage efficiency targets, quality of service (QoS), graceful degradation guidelines, and maximization of radio lifetime." (IEEE 2008a).

\section{Standards - A critical part of the evolution to CRS}

As conceptualized in Figure 1, the full potential of cognitive radio systems is dependent upon activities in the following areas:

- $\quad$ Economic feasibility based on business case scenarios:

- User requirements which drive new business cases which in turn drive the need for more spectrum (e.g., $4^{\text {th }}$ generation commercial wireless).

- New radio system technologies (e.g., ultra wideband, policy-based DSA radio systems, cognitive radio systems) and networks which utilize these radio system technologies - these new technologies open up new business opportunities.

- Technology Development:

- Responsive to new users requirements which frequently require significant new spectrum.

- Responsive to the need for more efficient use of the spectrum.

- Responsive to, and drives, new regulatory proceedings (e.g., DSA radios and networks, policy-based radio, software defined radio, ultra wideband radio (UWB), cognitive radio systems, etc.) and new questions within the ITU-R.

- Regulatory and policy changes:

- Regulatory changes that recognizes new requirements and new technologies.

- Regulatory changes for CRS and DSA are driven by need for a new paradigm for spectrum management to accommodate requirements for additional spectrum (e.g., the need for more spectrum for cellular systems to support wideband applications such as audio and video steaming).

As depicted in Figure 1, technical standards are a central part needed to bring the above components together to enable the fielding of CRS and DSA-based systems. One example of this is the development of IEEE 802 standards for TV white space as will be described later in this chapter.

Another example is the development of standards relative to advanced mobile telecommunications systems over the last several years. Clearly there is a business case as can be seen for the broadband applications. This required the consideration by the ITU-R and by national regulatory agencies for the identification of additional spectrum to support these new broadband applications. Technologies were developed to support these applications - these technologies were then standardized by the 3rd Generation Partnership Project (3GPP), IEEE, the ITU-R, and other standards organizations. Although this example does not specifically include CRS/DSA, these technologies are envisioned as being a part of the solution in future years for the continued evolution of mobile telecommunications requirements and the identification of spectrum to satisfy these ever increasing requirements. As will be described in later sections, the IEEE and the ITU-R have both been working on standards and reports relative to CRS/DSA for mobile telecommunication applications. 


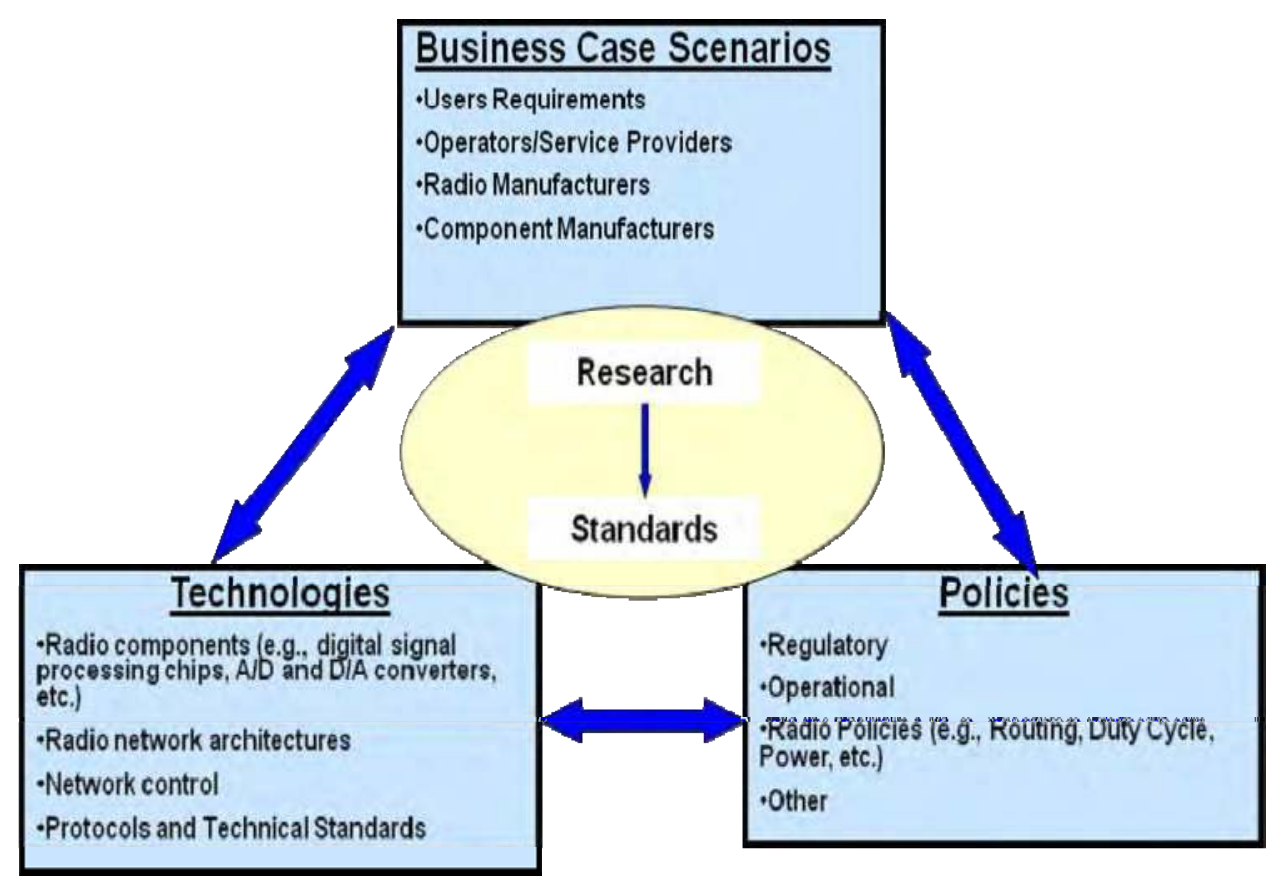

Fig. 1. Relationships between standards, technologies, policies and business cases for CRS and DSA

\section{International Telecommunication Union Radiocommunication Sector}

In our discussion of standards for CSA/DSA, a logical starting place is the International Telecommunication Union Radiocommunication Sector (ITU-R). The ITU-R is the sector of the ITU of primary relevance to this chapter because of its role as a primary global forum on the evolution of radio technologies and because of its decisions on spectrum allocations necessary for this evolution. Information on other sectors of the ITU as well as ITU-R may be found at www.itu.int.

The Radio Regulations (RR) is one of the most significant products of the ITU-R and is published in the 6 official languages of the ITU. (ITU-R, 2008) The Radio Regulations document is the international treaty governing the use of the radio-frequency spectrum and the geostationary-satellite and non-geostationary-satellite orbits. The RR incorporates the decisions of the World Radiocommunication Conferences (WRC) and includes ITU-R Recommendations incorporated by reference. The WRC is held every three to five years. The Radio Regulations (ITU-R, 2008) which are developed by the WRC and verified by signatures of the ITU Member States include:

- definitions of terms including allocation, allotment and assignment of frequencies

- the specific allocation of different frequency bands to different radio services;

- the mandatory technical parameters to be observed by radio stations, especially transmitters; 
- frequency assignment coordination procedures

- $\quad$ radio frequency interference regulations

- other administrative and operational procedures and provisions.

ITU-R Recommendations constitute a set of international technical standards developed by the Radiocommunication Sector of the ITU. They are the result of studies undertaken by Radiocommunication Study Groups and approved by ITU Member States (countries). Topics covered by ITU Recommendations and ITU Reports include:

- The use of a vast range of wireless services including those that mandate new telecommunication technologies such as CRS and DSA

- Spectrum management and the efficient use of the spectrum

- Radiowave propagation

- Systems and networks for wireless communications.

The use of ITU-R Recommendations and ITU-R Reports is not mandatory. However, since these documents are developed by technical experts from administrations (countries) around the world, communications systems operators, the telecommunications industry (including manufacturers and national and international standards organizations) and other organizations from all over the world that deal with radiocommunication matters, these documents are highly regarded and are implemented worldwide. Free online access to all current ITU-R Recommendations \& Reports is now provided to the general public: http://www.itu.int/pub/R-REC and http://www.itu.int/pub/R-rep

As will be discussed in the following sections, the ITU-R activities in regard to CRS and DSA include the following:

- $\quad$ WRC-12 Agenda Item 1.19 (ITU-R, 2007a).

- Resolution 956

- Agreement on definitions for cognitive radio systems and software defined radio.

- Development of ITU-R Reports and Recommendations on software defined radio, cognitive radio systems and related technologies.

\subsection{World Radiocommunication Conference Agenda Item 1.19 and Resolution 956}

The World Radiocommunication Conference (WRC) is organized by ITU to review and revise as necessary the ITU-R Radio Regulations. An important topic for the next WRC (January 2012 in Geneva) are issues related to software defined radio and CRS/DSA. One of the many Agenda Items of WRC-12 is Agenda Item 1.19:

"To consider regulatory measures and their relevance, in order to enable the introduction of software-defined radio and cognitive radio systems, based on the results of ITU- $R$ studies, in accordance with Resolution 956 of WRC-07." (ITU-R, 2007a).

ITU-R Resolution 956 states in part (ITU-R, 2007a):

"resolves to invite ITU-R

1. to study whether there is a need for regulatory measures related to the application of cognitive radio system technologies; 
2. to study whether there is a need for regulatory measures related to the application of softwaredefined radio.

Resolves further that WRC-11 consider the results of these studies and take the appropriate actions. ${ }^{1}$

The ITU-R studies relative to the potential need for additional regulatory measures is a critical step in the evolution of CRS. As previously discussed in regard to Figure 1, regulatory policies are one of the critical piece-parts in the evolution of CRS/DSA. Technologies which are developed to satisfy the business cases for CRS/DSA must be compliant with existing regulatory measures. The starting point for these regulatory measures is the ITU-R Radio Regulations. The following section provides information on the work of ITU-R Study Groups and Working Parties that are underway to satisfy Resolution 956. These results will be reviewed during WRC-12 as part of discussions relative to WRC-12 Agenda Item 1.19. Because of the complexity of the regulatory issues, it can reasonably be expected that these studies will be continued beyond WRC-12, i.e., into the next ITU-R Study Period and probably even beyond that although there may not be a continuation of the agenda item.

\subsection{Conference Preparatory Meetings}

There are two Conference Preparatory Meetings between WRCs; one is immediately after the conclusion of a WRC and is known as CPM1; the second CPM typically is a few months before the following WRC and is known as CPM2. CPM1 develops the program of study for the ITU-R Study Groups. CPM2 prepares a consolidated report to be used in support of the work of World Radiocommunication Conferences, based on:

- contributions from administrations and the Radiocommunication Study Groups concerning the regulatory, technical, operational and procedural matters to be considered by the following WRC;

- the inclusion, to the extent possible, of reconciled differences in approaches as contained in the source material, or, in the case where the approaches cannot be reconciled, the inclusion of the differing views and their justification. (ITU-R, 2007b).

\subsection{ITU-R study groups and working parties}

One of the major activities of the ITU-R is that associated with the ITU-R Study Groups and Working Parties. (ITU-R, 2010) The topics of study for ITU-R Study Groups are known as QUESTIONS. (ITU-R, 2011) These are documents that describe the scope and nature of studies that are assigned to Working Parties for study. This work ultimately results in one or more ITU-R Recommendations and ITU Reports. The QUESTIONS are assigned to Study Groups which in turn designate a Working Party within the Study Group to lead the effort of developing ITU-R Reports and Recommendations in response to the Question.

The primary Study Groups working on CRS studies are ITU-R Study Group 1 and ITU-R Study Group 5. Each of these Study Groups have been assigned QUESTIONS and work programs which guide the work of these Study Groups on CRS, DSA and related technologies.

${ }^{1}$ Note: The WRC that was originally scheduled for 2011 has been rescheduled for 2012 and is now referred to as WRC12. 


\subsubsection{ITU-R Study Group 1 - Spectrum Management}

The purpose of Study Group 1 (SG 1) is the development of "spectrum management principles and techniques, general principles of sharing, spectrum monitoring, long-term strategies for spectrum utilization, economic approaches to national spectrum management, automated techniques and assistance to developing countries in cooperation with the Telecommunication Development Sector." (ITU-R, 2010).

Working Party 1B (WP1B) is the Working Party under SG 1 that is responsible for the development of spectrum management methodologies and economic strategies. WP1B is responsible for Agenda Item 1.19 and is working on developing a response which will be sent from Study Group 1 to the CPM2 for WRC-12 in response to Resolution 956 from WRC07. This work within ITU-R is likely to continue well beyond WRC12 but is likely to be focused on additional studies that generate ITU-R Reports and ITU-R Recommendations rather than changes to the Radio Regulations.

\subsubsection{ITU-R Study Group 5 - Terrestrial Services}

ITU-R QUESTION 241-1/5 is entitled, "Cognitive Radio Systems in the Mobile Systems." And is assigned to ITU-R Working Party 5A (Land mobile service above $30 \mathrm{MHz}^{*}$ (excluding IMT); wireless access in the fixed service; amateur and amateur-satellite services). There are several parts of Question 241-1/5 (ITU-R, 2010). however, the key part of the Question is:

"How can cognitive radio systems promote the efficient use of radio resources?"

Although, WP5A has been assigned this Question, other Working Parties within Study Group 5 are also working on this topic. For example, ITU-R WP5D (International Mobile Telecommunications Systems) is working on an ITU-R Report entitled, "Cognitive radio systems specific for IMT systems." In the ITU-R, the term International Mobile Telecommunications (IMT) means a family of radio interfaces for mobile telecommunication services. The term is applied to $3^{\text {rd }}$ and $4^{\text {th }}$ generation commercial mobile telecommunication services.

A primary difference between the work of WP5A and WP5D on CRS is in their focus; whereas WP5D is focused on the IMT application of CRS, WP5A is focused primarily on non-IMT applications of CRS. Although the current reports being developed within ITU-R Working Parties 5A and 5D are likely to be completed in the near future, it is likely that work in this arena by these two working parties is likely to continue for several years. It is expected that ITU-R Recommendations and additional ITU-R Reports will be produced in addition to the ITU-R Reports currently being developed.

\subsection{Other ITU activities}

Radiocommunication Assemblies (RA) are responsible for the structure, program and approval of Radiocommunication studies. They are normally convened every three or four years and may be associated in time and place with World Radiocommunication Conferences (WRCs). Amongst other responsibilities, the Assemblies are responsible for:

- $\quad$ Suggesting suitable topics for the agenda of future WRCs;

- Approving and issuing ITU-R Recommendations developed by the Study Groups; 
- Assigning Questions to the Study Groups and setting the program of work for the Study Groups;

- $\quad$ Establishing and disbanding Study Groups according to need.

\section{IEEE standards development related to CRS and DSA}

A number of papers have been published in recent years that provide snapshots of international standards activities on CRS/DSA including those of the Institute of Electrical and Electronics Engineers (IEEE). (Sherman, et. al. 2008; Prasad, et. al., 2008; Granelli, et. al., 2010, and Stanislav, et. al. 2011) The branch of the IEEE that is responsible for standards development is the IEEE Standards Association (IEEE-SA). (http://standards.ieee.org/).

Under the IEEE-SA, there are standards boards associated with several IEEE Societies that have undertaken standards development relative to CRS/DSA. Examples are:

- IEEE Communications Society (ComSoc) Standards Board (CSSB)

- IEEE Electromagnetic Compatibility Society (EMC) Standards Development Committee (EMC SDCom).

- IEEE Computer Society Standards Activity Board.

The ComSoc Standards Board http://www.comsoc.org/about/standards/sponsors standards in communications and networking technologies and applications including the following technologies):

- $\quad$ Ad Hoc and Sensor Networks

- Cognitive Networks

- Communications and Information Security

- Communications Quality and Reliability

- Communications Software

- Communications Switching and Routing

- Computer Communications

- Communications Networks

- Internet Multimedia Communications

- Network Operations and Management

- Optical Communications and Networking

- $\quad$ Power Line Communications and Networking

- $\quad$ Radio, Satellite \& Space, and Wireless Communications and Networks

- $\quad$ Signal Processing and Communications Electronics

- Transmission, Access, and Optical Systems

More information on ComSoc standards activities may be found at:

http://committees.comsoc.org / standards/

The IEEE Electromagnetic Compatibility Society (EMC) also has keen interest in CRS/DSA because of its mission to develop and facilitate the exchange of scientific and technological knowledge in the discipline of electromagnetic environmental effects and electromagnetic compatibility. The EMC Society mission includes the development of electromagnetic compatibility standards and naturally includes interest in CRS/DSA because 
electromagnetic compatibility is at the heart of issues related to the ultimate deployment of CRS/DSA. For more information: http://www.emcs.org/standards/sdcomindex.html.

Two major efforts within the above IEEE Societies on CRS/DSA standards development are:

- The DySPAN Standards Committee (DySPAN-SC) which is sponsored by the Communications Society (ComSoc), and

- $\quad$ The IEEE 802 Committee which is sponsored by the Computer Society.

The IEEE EMC Society Standards Development Committee and the Communications Society Standards Board were the original sponsors of the IEEE standards organization that is now known as the DySPAN Standards Committee (DySPAN-SC) which is described in the next section.

The organizational relationships of key elements of the IEEE Standards Association involved with CRS/DSA standards development are depicted in Figure 2.

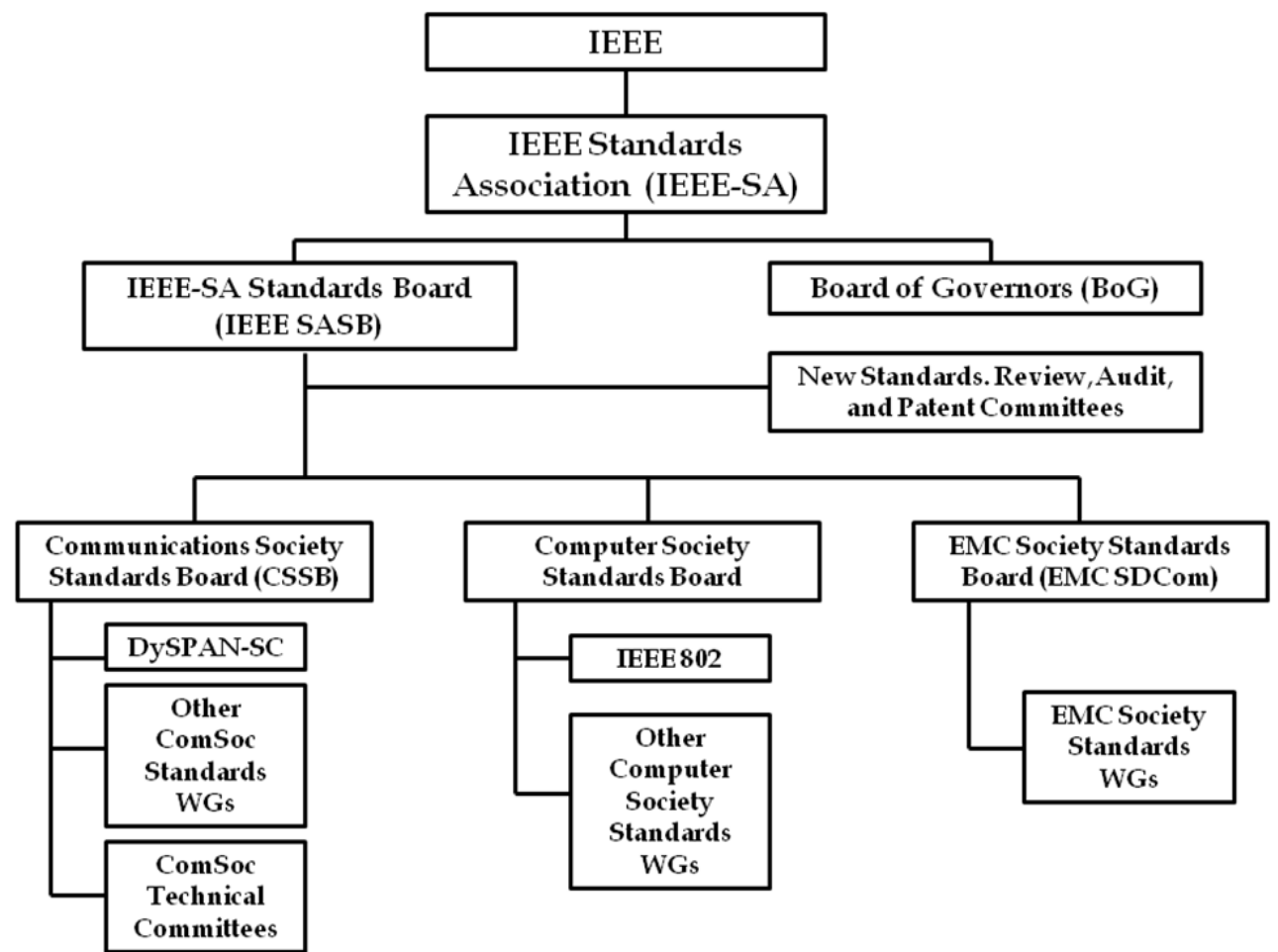

Fig. 2. IEEE Standards Organization. 


\subsection{IEEE DySPAN Standards Committee}

The DySPAN (Dynamic Spectrum Access) standards committee had its beginning in early 2005 jointly under CSSB and EMC. It was initially known as the 1900 Committee and later as the Standards Coordinating Committee 41 (SCC41). The scope of the DySPAN-SC includes the following:

- dynamic spectrum access radio systems and networks with the focus on improved use of spectrum,

- new techniques and methods of dynamic spectrum access including the management of radio transmission interference, and

- coordination of wireless technologies including network management and information sharing amongst networks deploying different wireless technologies.

Additional information on DySPAN-SC may be found at www.dyspan-sc.org.

Figure 3 is the DySPAN-SC organization chart. The detailed technical work of the IEEE DySPAN-SC is performed by the IEEE 1900.x Working Groups (WGs) which are shown in the figure and which are described in the following subsections. Several of the 1900.x WGs have completed baseline standards and will be developing follow-on standards that are extensions of the baseline standards. Therefore, it is expected that each of these IEEE 1900.x WGs will be in existence for many years.

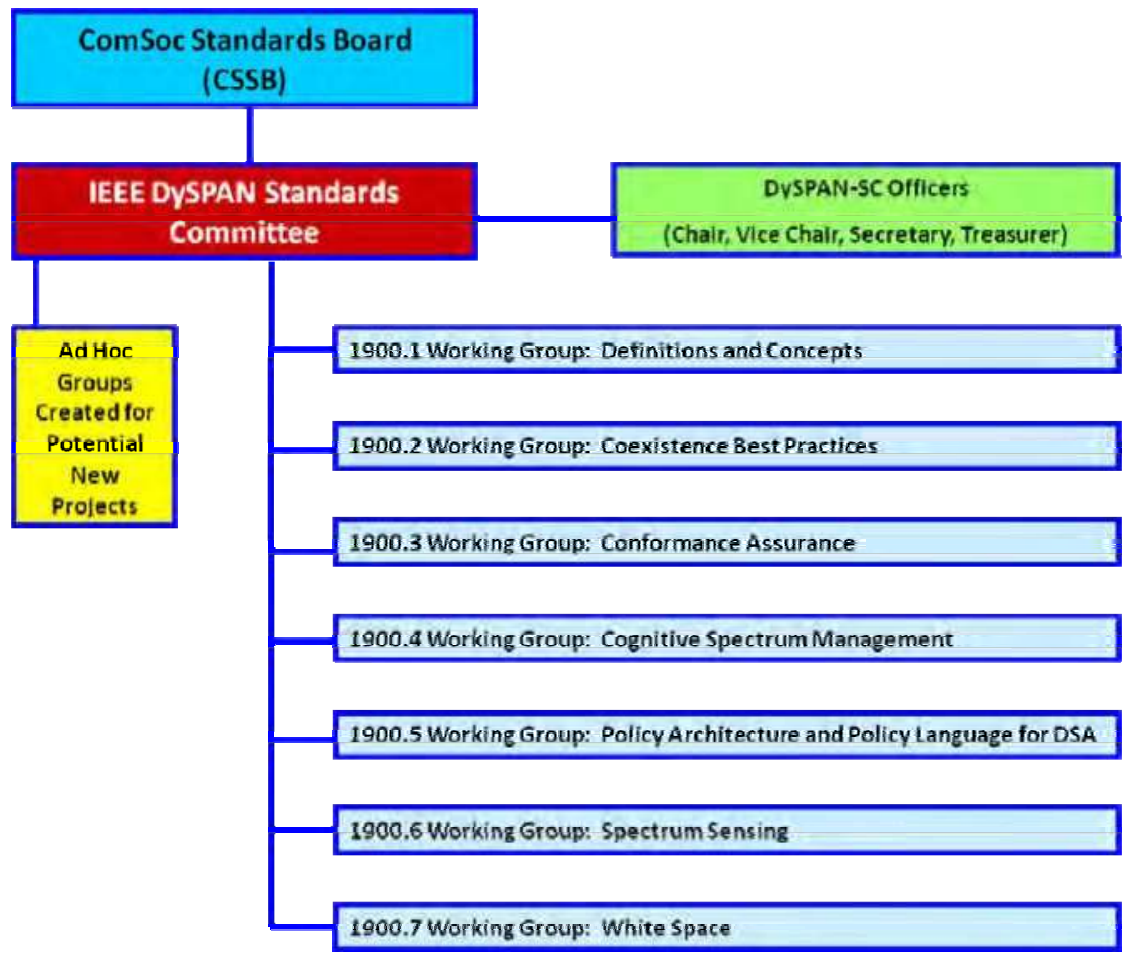

Fig. 3. Organizational chart of the DySPAN Standards Committee showing working groups 


\subsubsection{IEEE 1900.1 Working Group}

The IEEE 1900.1 WG is responsible for DSA definitions and concepts and published its first standard in 2008. This standard provides definitions and explanations of key concepts in the fields of spectrum management, cognitive radio, policy-defined radio, adaptive radio, software-defined radio (SDR), and related technologies. The standard goes beyond simple, short definitions by providing amplifying text that explains these terms in the context of the technologies that use them. The document also describes how these technologies interrelate and create new capabilities while at the same time providing mechanisms supportive of new spectrum management paradigms such as CRS and DSA. (IEEE 2008a).

The body of this standard is "normative" meaning that the material is a required part of the standard. Because of the complexity of the technology, the 1900.1 Standard provides amplifying material in "informative" annexes. Although these "informative" annexes are not a required part of the standard, these annexes are helpful to users of the standard in fully understanding the meaning of the "normative" definitions. A key annex is the annex that describes the relationship of terms such cognitive radio, software defined radio, software-controlled radio, intelligent radio, policy-based radio, and reconfigurable radio.

The IEEE 1900.1 Standard TM defines "cognitive radio" (CR) and "cognitive radio network" but does not define "cognitive radio system" which is a term defined by the ITU-R and the US National Telecommunications and Information Administration and provided earlier in this chapter. (ITU 2009 and NTIA 2011). The IEEE and ITU-R definitions for CR and for CRS respectively are consistent however. The note in the IEEE definition for CR states the cognitive functionality typically associated with a cognitive radio is beyond the functionality normally associated with a "radio." The key is having a common understanding of what is meant by "radio."

The IEEE 1900.1 Standard TM defines "radio" as being a "technology for wirelessly transmitting or receiving electromagnetic radiation to facilitate transfer of information." In this context, it is important to understand and differentiate between "radio" and "radio systems." Radio systems include software control of the radio proper. This radio control may include cognitive and adaptive features. The IEEE 1900.1 Standard TM provides figures and text useful in the understanding of these aspects of cognitive radio, cognitive radio systems and radio control mechanisms.

Figure 4 is an illustration of a basic concept derived from the concepts provided in the IEEE 1900.1 Standard TM. The figure illustrates what is meant by software control of a software defined radio. A software defined radio is defined by IEEE 1900.1 Standard TM as:

A type of radio in which some or all of the physical layer functions are software controlled. Software control is the use of software processing within the radio system or device to select the parameters of operation.

The parameters of operation include the radio frequency (rf), the modulation, the transmitted power level, etc., i.e., the parameters that one typically associations with radio operation. In a software defined radio, some or all of these radio operational parameters may be implemented in software and may be changed by a real-time software control mechanism. The software control mechanisms may be very complex and may include cognitive and adaptive functionality that responds in real-time to a changing rf 
environment, changing operational policies, changing location, etc. More will be said in Section 5.1.5 about the policy-based radio control mechanism.

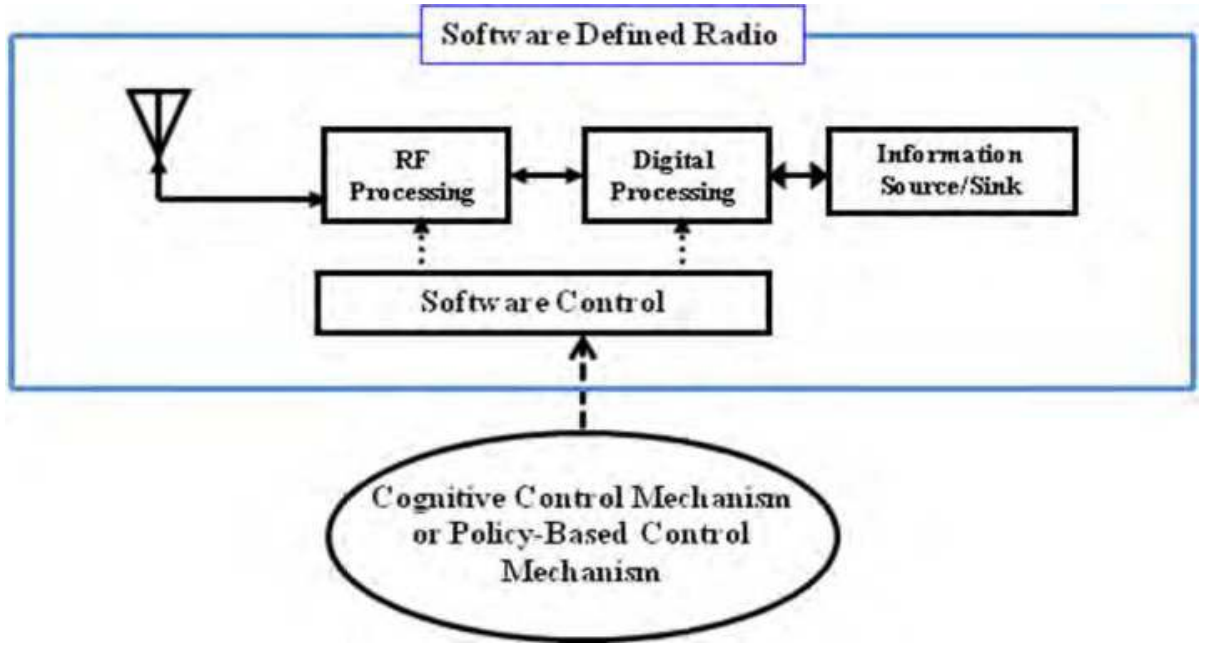

Fig. 4. Illustration of software control of software defined radio

\subsubsection{IEEE 1900.2 Working Group}

The IEEE 1900.2 WG has produced a standard which provides technical guidelines for analyzing the potential for coexistence or, by way of contrast, interference between radio systems operating in the same frequency band or between different frequency bands. (IEEE, 2008b). Additional work in this arena is needed and is likely to be conducted by one or more of the IEEE 1900.x WGs that operate under the DySPAN-SC.

\subsubsection{IEEE 1900.3 Working Group}

The IEEE 1900.3 WG was dissolved prior to the completion of any standard. It was chartered to produce a standard which would:

- $\quad$ specify techniques for testing and analysis to be used during compliance and evaluation of radio systems with dynamic spectrum access capability, and which would

- $\quad$ specify radio system design features that simplify the evaluation challenge.

The potential for deployment of CRS/DSA systems may be affected by the ability of regulatory agencies and industry stakeholders to verify that a system conforms to applicable technical and policy requirements. Thus, the future reactivation of the IEEE 1900.3 WG is needed. The initiation of the IEEE 1900.3 Project in 2006 was undoubtedly premature. The topic of what kind of new regulation, if any, is being vigorously debated within the ITU-R and within various national regulatory bodies. It can be reasonably expected that the IEEE 1900.3 WG activities will be resurrected as the ITU-R progresses its work on Agenda Item 1.19 to consider regulatory requirements for CRS and SDR and as national regulatory bodies take action on this critical issue. 


\subsubsection{IEEE 1900.4 Working Group}

The IEEE 1900.4 WG has published a standard which defines the building blocks comprising

- network resource managers,

- device resource managers, and

- $\quad$ the information to be exchanged between the building blocks. (IEEE 2009)

This standard enables coordinated network-device distributed decision making which will aid in the optimization of radio resource usage, including spectrum access control, in heterogeneous wireless access networks. The standard is limited to the architectural and functional definitions at a first stage. The corresponding protocols definition related to the information exchange is now being addressed during a second stage of IEEE 1900.4 WG activities.

The purpose of the IEEE 1900.4 project is to improve overall composite capacity and quality of service of wireless systems in a multiple Radio Access Technologies (RATs) environment, by defining an appropriate system architecture and protocols which will facilitate the optimization of radio resource usage.

In addition to the baseline IEEE 1900.4 Standard TM, an amendment to this standard has recently been completed. (IEEE 2011a). This amendment modifies the IEEE 1900.4 standard to enable mobile wireless access service in white space frequency bands without any limitation on used radio interface (physical and media access control layers, carrier frequency, etc) by defining additional components of the IEEE 1900.4 system. This amendment is integrated into the baseline standard.

An additional project of the IEEE 1900.4 working group is the P1900.4.1 project which enhances the baseline standard by providing detailed specification of interfaces and service access points defined in the baseline standard, This enables distributed decision making in heterogeneous wireless networks and the use of context information for this decision making.

\subsubsection{IEEE 1900.5 Working Group}

The IEEE 1900.5 WG has produced a standard which defines a vendor-independent set of policy-based control architectures and corresponding policy language requirements for managing the functionality and behaviour of dynamic spectrum access networks. This standard also defines the relationship of policy language and architecture to the needs of at least the following constituencies: the regulator, the operator, the user, and the network equipment manufacturer. (IEEE 2012).

Figure 5 is a simplified user interpretation of the architecture provided in the IEEE 1900.5 Standard TM for a policy-based DSA radio system (PBDRS) functional architecture. This figure may be viewed as an expansion of the more general architecture provided in Figure 4. The additional details are those involved in the software control mechanism and consist of the system strategy reasoner (SSR), the policy enforcer (PE) and the policy conformance reasoner (PCR). Essentially these components are designed to ensure compliance by the PBDRS to policies which may change depending on many parameters such as user requirements, rf environment, geographical location, etc. Some of these changes may be 
real-time; for example changes to the CRS operations due to changes in the rf environment. This type of capability is needed to reach the ultimate goal of more efficient use of the spectrum.

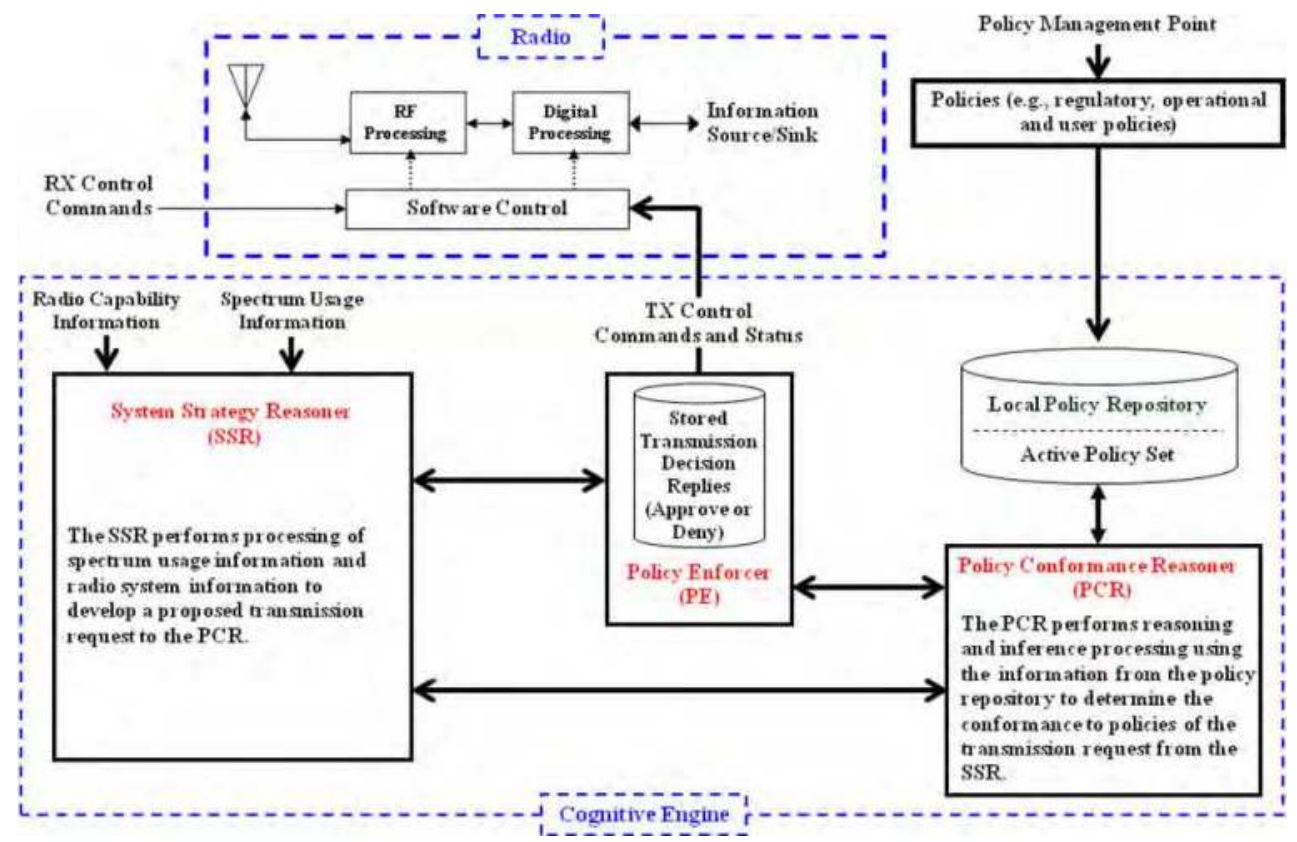

Fig. 5. Simplified interpretation of IEEE 1900.5 policy-based DSA radio system architecture.

The PCR, SSR and the PE represent functionalities that may be distributed, i.e., this functionality may not reside within a single radio device or even within a radio node. This is the reason for the emphasis on the system and network and not just the radio per se (meaning not just the receiver and transmitter aspect).

The policies that come from the policy management point (PMP) may be regulatory policies, operational policies or even user policies. The input to the PMP may be human readable policies that are converted into digital policies that are machine readable.

The IEEE 1900.5 Standard TM (IEEE 2012) describes the communications between the SSR, PE and PCR which are shown in Figure 5; however the standard does not specify the details of these interfaces. One follow-on standard to the base standard will specify these interfaces in detail. The detailed specification of these interfaces may be helpful to regulatory agencies that will be addressing certification issues for these types of radios which can be viewed as a new paradigm. Information from the research community including ComSoc Technical Committees such as the Technical Committee on Cognitive Networks will be helpful in the development of this follow-on standard. 
In addition to the functional architecture just described, the IEEE 1900.5 Standard TM also describes requirements for a policy language to be used by the PCR. This language will be a declarative language. This type of language expresses what is to be accomplished rather than how it is to be accomplished. This is in contrast to languages such as $\mathrm{C}, \mathrm{C}^{++}$, and Java which are imperative or procedural languages that manipulate the state of the program through an executed sequence of commands specified by a control structure. A follow-on to the IEEE 1900.5 Standard TM will provide a detailed specification of this policy language based on the requirements listed in the base standard.

\subsubsection{IEEE 1900.6 Working Group}

The IEEE 1900.6 WG has produced a standard which defines the information exchange between spectrum sensors and their clients in radio communication systems. The logical interface and supporting data structures used for information exchange are defined abstractly without constraining the sensing technology, client design, or data link between the sensor and client. The purpose of this standard is to define spectrum sensing interfaces and data structures for DSA systems and other advanced radio communications systems that will facilitate interoperability between independently developed devices and thus allow for separate evolution of spectrum sensors and other system functions. (IEEE 2011b).

The standard contains system models that allow for multiple sensors and for multiple cognitive engines or data archives. In other words, the sensor data acquisition can be distributed with multiple sensors, the sensor processing can be distributed with multiple cognitive engines, and the sensor data storage can be distributed with multiple data archives.

Sensing of the rf environment is a critical part of the original CRS/DSA concept. It is well recognized, however, that sensing of the rf environment is itself not always sufficient to have a realistic picture of the rf environment. For instance, sensing will not detect the usage of spectrum by some passive users of the spectrum such as radio astronomy. Therefore, sensing may need to be supplemented with other sources of information on the rf spectrum usage such as data bases. Chouinard (2010) discusses the practical limits to rf sensing and specifically notes that although it is a good approach to be considered in general for cognitive radio, it has been a challenge for us in the TV white space bands.

\subsubsection{IEEE 1900.7 Working Group}

In June 2011, the IEEE 1900.7 WG commenced work on a standard that specifies a radio interface including medium access control (MAC) sublayer(s) and physical (PHY) layer(s) of white space dynamic spectrum access radio systems supporting fixed and mobile operation in white space frequency bands, while avoiding causing harmful interference to incumbent users in these frequency bands. The standard provides supports the IEEE 1900.4 Standard TM, the IEEE 1900.4a Amendment (IEEE (2011a) for white space management, and the IEEE 1900.6 Standard TM to obtain and exchange sensing related information (spectrum sensing and geolocation information).

The IEEE P1900.7 Standard will enable the development of cost-effective, multi-vendor white space dynamic spectrum access radio systems capable of interoperable operation in white 
space frequency bands on a non-interfering basis to incumbent users in these frequency bands. This standard facilitates a variety of applications, including the ones capable to support high mobility, both low-power and high-power, short-, medium, and long-range, and a variety of network topologies. This standard is a baseline standard for a family of other standards that are expected to be developed focusing on particular applications, regulatory domains, etc.

One result of TV broadcasting systems being upgraded from analog to digital technology is that it frees up channels under certain conditions (e.g., that the channel is not being used by licensed wireless microphones). Unlike the white space work in IEEE 802 which is discussed later in this chapter, the IEEE 1900.7 WG is not focused on the TV white space bands (TVWS). The intent of 1900.7 is the development of a family of standards that could be used for any portion of the spectrum having white space, i.e., spectrum that is not being utilized.

\subsection{IEEE 802}

The IEEE-802 Local Area Network/Metropolitan (LAN/MAN) Standards Committee (LMSC) develops LAN/MAN standards primarily for the lowest 2 layers of the ISO Reference Model for Open Systems Interconnection (OSI). The first meeting of the IEEE "Local Area Network Standards Committee", Project 802, was held in February of 1980. Like the DySPAN-SC, there is strong international participation in IEEE 802. Some IEEE 802 Standards are published as International Standards Organization (ISO) Standards. (IEEE 802, 2008). Additional general information about IEEE 802 may be found at: http://www.ieee802.org and http://standards.ieee.org/about/get/

Like the DYSPAN-SC, the primary work of IEEE 802 is performed in the IEEE 802 Working Groups. Only the IEEE 802 WGs that are involved in CRS/DSA standards are briefly discussed in the following subsections. Information on all of the IEEE 802 WGs may be found at: http://www.ieee802.org/dots.shtml

The work of the 802 WGs is a practical example of the concepts illustrated in Figure 1 regarding relationships between standards development, technology, and regulatory policies. The work of these WGs is a direct result of decisions made by regulatory agencies regarding TV white space. (FCC 2004, FCC 2008, FCC 2011). Clearly there are business case questions related to this work - new LAN business case potential for the IEEE 802.11af WG and new WRAN business cases for the IEEE 802.22 WG for example.

The CRS/DSA activity within IEEE 802 is the direct result of regulatory actions taken by the US Federal Communications Agency (FCC) and other national regulatory bodies. In the US, this started in 2002 by the FCC issuance of a Notice of Inquiry (NOI) to explore the possibility of allowing access to the TV broadcast bands for license-exempt devices on a non-interfereing basis for operation in VHF and UHF TV bands. (FCC, 2004). The IEEE 802 views this FCC action and the resulting decisions (FCC, 2008 and FCC 2011) as a potential business oportunity to:

- $\quad$ enhance IEEE 802.11 LAN functionality

- use of the TV white space bands for rural area broadband coverage using the IEEE 802.22 Wireless Regional Area Networks.

These IEEE 802 standards development efforts are discussed briefly in the following subsections. 


\subsubsection{IEEE 802.11 Wireless LAN Working Group}

The IEEE 802.11 WG is well known for the family of IEEE 802.11 Standards. http://www.ieee802.org/11/. In December 2009, a Project Approval Request (PAR) was approved by the IEEE SASB to develop a new standard that will be part of the IEEE 802.11 family of standards. The title of this proposed standard is:

"Standard for Information Technology - Telecommunications and Information Exchange Between Systems - Local and Metropolitan Area Networks - Specific Requirements - Part 11: Wireless LAN Medium Access Control (MAC) and Physical Layer (PHY) Specifications; Amendment: TV White Spaces Operation."

This standard will be known as IEEE 802.11af. The main difference between this standard and other members of the well known IEEE 802.11 family of standards is that IEEE802.11af will be a based on cognitive radio for operation in the TV White Spaces. The TV White Spaces is spectrum already allocated to the TV broadcasters but is not being used. The only additional prerequisite for IEEE 802.11af in the US, is the need for a database that maintains data about used frequencies in the TV band.

With the global transition to digital TV, sub-Gigahertz rf spectrum is becoming available. Much of this spectrum is for unlicensed, license exempt and/or lightly licensed use. The IEEE 802.11af standard will make the necessary MAC and PHY changes to enable 802.11 products to take advantage of this additional spectrum. The purpose of the IEEE 802.11af standard is to allow 802.11 wireless networks to be used in the TV white space. The scheduled completion date for the IEEE 802.11af Standard is during 2013. http://www.ieee802.org/11/Reports/802.11_Timelines.htm

\subsubsection{IEEE 802.16 Broadband Wireless Access WG}

The IEEE 802.16 Working Group on Broadband Wireless Access Standards develops standards and recommended practices to support the development and deployment of broadband Wireless Metropolitan Area Networks. This WG does not have projects that specifically develop CRS/DSA standards. However CRS/DSA technology may be incorporated into the standards developed by the IEEE 802.16 WG such as the wireless metropolitan area network (MAN) air interface which has been approved by ITU-R Working Party 5D as an IMT Advanced (Internal Mobile Telecommunications) air interface. http://ieee802.org/16/index.html

\subsubsection{IEEE 802.18 - Radio Regulatory Technical Advisory Group}

The IEEE 802.18 Radio Regulatory Technical Advisory Group (RR-TAG) supports the work of the IEEE 802 LMSC and the IEEE 802 WGs including IEEE 802.11 (WLAN), IEEE 802.15 (WPAN), IEEE 802.16 (WMAN), IEEE 802.20 (Wireless Mobility), IEEE 802.21 (Handoff/Interoperability Between Networks), and IEEE 802.22 (WRAN). This support includes actively monitoring and participating in radio regulatory matters worldwide as an advocate for IEEE 802. One example is representing IEEE in the ITU-R.

http://ieee802.org/16/index.html 


\subsubsection{IEEE 802.19 - Wireless Coexistence WG}

The IEEE 1900.1 WG

- develops standards for coexistence between wireless standards of unlicensed devices, and

- reviews coexistence assurance (CA) documents produced by other IEEE 802 WGs developing new wireless standards for unlicensed devices.

The IEEE 802.19.1 subgroup is in the process of developing a standard entitled:

Standard for Information Technology - Telecommunications and Information Exchange Between Systems - Local and Metropolitan Area Networks - Specific Requirements - Part 19: TV White Space Coexistence Methods

The purpose of the standard is to enable the family of IEEE 802 Wireless Standards to most effectively use TV White Space by providing standard coexistence methods among dissimilar or independently operated TV band devices (TVBD) networks and dissimilar TVBDs. This standard addresses coexistence for IEEE 802 networks and devices and will also be useful for non IEEE 802 networks and TVBDs. Completion in 2012.

http://ieee802.org/19/index.html

\subsubsection{IEEE 802.22 - Wireless Regional Area Networks WG}

The IEEE 802.22 Working Group on Wireless Regional Area Networks (WRANs) is responsible for developing standards for a cognitive radio-based PHY/MAC/air interface for use by license-exempt devices on a non-interfering basis in spectrum that is allocated to the TV Broadcast Service.

The IEEE 802.22 WRAN Standard uses cognitive radio techniques to allow sharing of geographically unused spectrum allocated to the television broadcast service, on a noninterfering basis. The goal is to bring broadband access to hard-to-reach, low-populationdensity areas which are typical of rural environments. A particularly difficult problem is that of ensuring that there is no interference to low-powered licensed devices such as wireless microphones.

IEEE 802.22 Provides Three Mechanisms for Incumbent Protection:

- Sensing

- Database Access

- Specially Designed Beacon

One or more protection mechanisms can be adopted based on the regulatory domain requirements.

Stevenson, et.al (2009) and Mody and Chouinard (2010) provide overviews of the IEEE 802.22 standards work.

Projects have been approved by IEEE SASB to develop the following standards:

- IEEE 802.22: Standard for Information Technology -Telecommunications and information exchange between systems - Wireless Regional Area Networks (WRAN) Specific requirements - Part 22: Cognitive Wireless RAN Medium Access Control 
(MAC) and Physical Layer (PHY) specifications: Policies and procedures for operation in the TV Bands

- IEEE 802.22.1: Standard to enhance harmful interference protection for low power licensed devices operating in TV Broadcast Bands

- IEEE 802.22.2: Information Technology - Telecommunications and information exchange between systems - Wireless Regional Area Networks (WRAN) - Specific requirements - Part 22.2: Recommended Practice for the Installation and Deployment of IEEE 802.22 Systems

- IEEE 802.22a: Amendment to IEEE Standard for Wireless Regional Area Networks Part 22: Cognitive Wireless RAN Medium Access Control (MAC) and Physical Layer (PHY) specifications: Policies and procedures for operation in the TV Bands. Management and Control Plane Interfaces and Procedures and Management Information Base Enhancements.

The IEEE 802.22 baseline standard and IEEE 802.22.1 standard have been published. (IEEE 802.22, 2010) (IEEE 802.22, 2011).

\section{Other activities related to standards for CRS and DSA}

There are numerous other organizations that are involved either directly or indirectly in the development of national and international standards for CRS, DSA and related technologies. It is not possible in this short chapter to discuss all of these organizations. One source that provides a list of standards setting organizations (SSOs) is ConsortiumInfo.org. For example, information on SSOs for telecommunications may be found at:

http:/ / www.consortiuminfo.org/links/linkscats.php?ID=26

It is important to realize that some standards such as those produced by the IEEE and ITU-R are not implemented directly. Instead, numerous alliances have been created to develop more specific documents that are based on what comes out of the IEEE. Examples of this are:

- The WiFi Alliance creates specifications based on IEEE 802.11 Standard.

- The WiMAX Forum creates specifications based on the IEEE 802.16 Standard

- The WRAN Alliance creates specifications based on the IEEE 802.22 Standard and other standards.

- The 3GPP and other organizations create specifications that are responsive to ITU-R Recommendations. These specifications are then adopted by the ITU-R.

The following subsections briefly describe some other organizations involved in CRS/DSA and related technologies. The list is not considered to be all inclusive.

\subsection{European Telecommunications Standards Institute}

The European Telecommunications Standards Institute (ETSI) produces globally-applicable standards for information and communications technologies (ICT), including fixed and mobile telecommunications and internet technologies. ETSI is officially recognized by the European Union as a European Standards Organization. ETSI has more than 700 member organizations from 62 countries in 5 continents. 
More information made be found at: http://www.etsi.org/WebSite/homepage.aspx

Mueck (2010a) provides a summary of ETSI work on reconfigurable radio systems (RRS), SDR and CR standards and the European Regulatory Framework. The cognitive radio efforts within EYSI are focused on

- a cognitive pilot radio channel, and

- a functional architecture for management and control of RRS.

The ETSI RRS Technical Committee (TC) develops studies and standards complementary to the IEEE DySPAN-SC and IEEE 802 standards development activities. The focus of the ETSI is on:

- $\quad$ SDR standards

- CR/SDR standards addressing the specific needs of the European Regulatory Framework,

- CR/SDR TV white space standards adapted to the digital TV signal characteristics in Europe.

The ETSI RRS TC comprises four WGs (Mueck, 2010a).:

- WG1 focuses on system aspects and develops proposals from a system aspects point of view for a common framework in RRS TC.

- WG2 focuses on SDR technology with a particular interest in radio equipment architecture and proposes common reference architectures for SDR/CR radio equipment.

- WG3 focuses on cognitive management and control including a functional architecture for radio resource management and a cognitive pilot channel.

- WG4 focuses on public safety and collects and defines the related RRS requirements from relevant stakeholders in the public safety and defense domain.

These ETCI RRS TCs have completed numerous studies regarding CRS, SDR, and CR as the result of several Europe Union funded programs. One focus now in ETSI RRS TC is on utilizing the results of these research studies in developing appropriate normative standards. Mueck (2010b) provides additional information.

\subsection{Ecma International}

Ecma International was originally founded as the European Computer Manufacturers Association; however the name is now officially Ecma International without reference to the original ECMA acronym. Since 1961 Ecma International and its predecessor organization have developed international standards in information technology and telecommunications. More than 370 Ecma Standards and numerous Technical Reports have been published, many of which have also been adopted as international standards and/or technical reports.

Ecma International has published a standard on medium access control layer (MAC) and physical layer (PHY) specifications for operation in TV white space. (Ecma, 2009). This standard specifies MAC and PHY for personal/portable cognitive wireless networks operating in TV bands. The standard also provides specifications for higher layer protocols. It specifies a number of incumbent protection mechanisms available to meet regulatory requirements. 
More information may be found at:

http://www.ecma-international.org/activities/index.html

\subsection{IEEE ComSoc technical committees}

The IEEE Communications Society has more than 24 technical committees. These technical committees define and implement the technical directions of ComSoc. All ComSoc Members are encouraged to participate in one or more of these technical committees. These committees usually meet twice a year at major conferences. Throughout the year, these committees also play a major role in determining which events (conferences, workshops, etc.) are technically co-sponsored by ComSoc.

The ComSoc Technical Committees may be tasked by ComSoc Working Groups to provide assistance on specific technical issues that may arise during the development of a standard being developed under ComSoc Standards Board sponsorship. Of particular interest to standards activities responsible for standards on CRS/DSA are the Cognitive Networks, Radio, and Wireless Technical Committees.

More information may be found at: http://www.comsoc.org/about/committees/Technical

\subsection{Wireless Innovation Forum}

The Wireless Innovation Forum ${ }^{\mathrm{TM}}(\mathrm{WInF})$ is a non-profit corporation whose predecessor organization was started in 1996. Formerly known as the SDR Forum, the WInF is dedicated to driving technology innovation in commercial, civil, and defense communications worldwide. The focus of the WInF is on SDR, CR, and DSA. The Forum has produced many technical reports on these topics. The Forum is not an internationally accredited standards development organization. However, the technical reports developed by the Forum have been input to standards organizations such as IEEE DySPAN-SC and ITU-R. For example, the Forum contributed many inputs to the development of the IEEE 1900.1 Standard TM.

More information on the Wireless Information Forum may be found at: http://www.wirelessinnovation.org/page/About_the_Forum

\subsection{WRAN Alliance}

The Wireless Regional Area Network (WRAN) Alliance promotes products and services based on wireless telecommunications standards for broadband services using TV band frequencies.

The WRAN Alliance has the objective of promoting standards that will permit the provision of broadband connectivity at reasonable cost in rural areas and in developing areas. This is viewed by the WRAN Alliance as a means bridging the digital divide.

The WRAN Alliance is initially focusing on promoting the use of the IEEE 802.22 Standard on Wireless Regional Area Networks, in variety of different regulatory domains and for a variety of different use cases. Use cases include but are not limited to the following:

- broadband access over large distances,

- broadband access for remote and rural areas, 
- long-range backhaul,

- smart grid and critical infrastructure monitoring,

- defense,

- homeland security,

- healthcare, and

- small office.

More information on WRAN Alliance may be found at: www.wranalliance.org/

\subsection{Wireless World Research Forum}

The objective of the Wireless World Research Forum (WWRF) is to formulate visions on strategic future research directions in the wireless field, among industry and academia, and to generate, identify, and promote research areas and technical trends for mobile and wireless system technologies. The WWRF is not an internationally accredited standards development organization. However, the results of the WWRF research have significant impact on the direction of standards development in many international standards development organizations. The WWRF has one group focused on cognitive networks for wireless internet and another on spectrum issues.

More information may be found at: http://www.wireless-world-research.org/

\section{Conclusion and future work}

This chapter has provided a roadmap to international standardization activities for cognitive radio systems, DSA systems and related technologies. Numerous standards have already been published by several standards bodies. In addition, there are many technical reports related to standards that have developed and are continuing to be published by a variety of organizations such as the ITU-R, WWRF, ETSI, and the Wireless Innovation Forum. Although these technical reports are not standards per se, they nevertheless provide the foundation for future standards development.

To a certain extent this roadmap to CRS/DSA standards development could be considered to be a snapshot in time. However, the information on organizations and standards provided herein is essentially a description of the foundations of the CRS/DSA standardization effort. It is anticipated that all of the organizations mentioned herein will still be active in CRS/DSA standardization for at least the next decade. Furthermore, it is anticipated that the specification standards mentioned herein are in most cases baseline standards that are the foundation for work that will be ongoing for the next decade or more.

The CRS/DSA standardization effort is very complex because ultimate success of this technology is dependent on the efforts of the regulatory, technical research, standards development, and business communities. Although these communities are all part of the effort for ultimately successfully fielding these technologies, these communities work independently.

A driving force is the need to more efficiently utilize the rf spectrum. Thus, additional regulatory action may be needed to free up more spectrum for CRS and DSA -- the FCC rulings related to TV white space provide an example of what may be forthcoming. But clearly, these 
types of rulings can be controversial. The research community can come up with technology proposals for better utilization of the spectrum, but it must be within regulatory boundaries. Standards development organizations can develop new standards that incorporate results from the research community, but these standards also must stay within the regulatory boundaries. But the regulatory boundaries can be changed depending on the influence of industry on regulators - this influence is the result of business cases for the use of spectrum.

Finally, it can be expected that the ITU-R, IEEE DySPAN-SC, IEEE 802 and many other organizations identified in this chapter will continue their standardization efforts for at least another decade. Regulatory boundaries, technology, business requirements will all evolve during this period of time and will be driven by the need to more efficiently utilize the spectrum.

\section{References}

Chouinard, G. (2010). RF Sensing in the TV White Space, 8th Conference on Communications Networks and Services Research (CNSR2010) held at McGill University, 12-14 May 2010.

Ecma (2009). MAC and PHY for Operation in TV White Space, ECMA-392, available at: http://www.ecma-international.org/publications/standards/Ecma-392.htm

FCC (2004). Notice of Proposed Rule Making, In the Matter of Unlicensied Operation in the TV Broadcast Bands, US FCC Docket 04-186, May 25, 2004.

FCC (2010). Second Report and Order and Memorandum or Opinion and Order, in the Matter of Unlicensed Operation in the TV Broadcast Bands Additional Spectrum for Unlicensed Devices Below $900 \mathrm{MHz}$ and in the $3 \mathrm{GHz}$ Band, Oct. 18, 2006.

FCC (2011). Unlicensed Operation in the TV Boradcast Bands, Additional Spectrum for Unlicnsed Devices Below $900 \mathrm{MHz}$ and in the $3 \mathrm{Ghz}$ Band, FCC DA 11-131, January 26, 2011.

Granelli, F., Pawelczak, P., Prasad, V., Subbalakshmi, K., Chandramouli, R., Hoffmeyer, J., and Berger, H., (2010). Standardization and Research in Cognitive and Dynamic Spectrum Access Networks: IEEE SCC41 Efforts and Other Activities, IEEE Comm. Mag., January 2010.

IEEE (2008a). IEEE Standard Definitions and Concepts for Dynamic Spectrum Access: Terminology Relating to Emerging Wireless Networks, System Functionality, and Spectrum Management.

IEEE (2008b). IEEE Recommended Practice for the Analysis of In-Band and Adjacent Band Interference and Coexistence Between Radio Systems.

IEEE (2009). IEEE Standard for Architectural Building Blocks Enabling Network-Device Distributed Decision Making for Optimized Radio Resource Usage in Heterogeneous Wireless Access Networks.

IEEE (2011a). 1900.4a, Standard for Architectural Building Blocks Enabling Network-Device Distributed Decision Making for Optimized Radio Resource Usage in Heterogeneous Wireless Access Networks - Amendment: Architecture and Interfaces for Dynamic Spectrum Access Networks in White Space Frequency Bands, 16 September 2011.

IEEE (2011b). IEEE Standard for Spectrum Sensing Interfaces and Data Structures for Dynamic Spectrum Access and Other Advanced Radio Systems.

IEEE (2012). IEEE Standard Policy Language Requirements and System Architectures for Dynamic Spectrum Access Systems. (Approved for final publication in January 2012). 
IEEE 802 (2008) Overview and Guide to the IEEE 802 LMSC http:/ / www.ieee802.org/IEEE802-LMSC-Overview-and-Guide-01.pdf

IEEE 802.22 (2010), IEEE Standard for Informatin Technology - Telecommunications and information exchange between systems - Local and metropolitan area networks Specific requirements: Part 22.1 Standard to Enhance Harmful Interference Protection for Low-Power Licensed Device Operation in the TV Broadcast Bands, IEEE Std 802.22.1TM-2010, 1 November 2010

IEEE 802.22 (2011). Standard for Information Technology -Telecommunications and information exchange between systems - Wireless Regional Area Networks (WRAN) - Specific requirements - Part 22: Cognitive Wireless RAN Medium Access Control (MAC) and Physical Layer (PHY) specifications: Policies and procedures for operation in the TV Bands, July 2011

ITU-R (2007a). WRC-12 Agenda Item 1.19: Software Defined Radio (SDR) and Cognitive Radio Systems (CRS) http://www.itu.int/ITU-R/information/promotion/eflash/4/article5.html

ITU-R (2007b) RESOLUTION ITU-R 2-5, Conference Preparatory Meeting, (1993-1995-19972000-2003-2007)

ITU-R (2008). International Telecommunication Union Radio Regulations, http://www.itu.int/pub/R-REG-RR/en

ITU-R (2009). Definitions of Software Defined Radio (SDR) and Cognitive Radio System (CRS), ITU-R Recommendation SM 2152

ITU-R (2010). ITU-R Study Groups, http://www.itu.int/dms_pub/itu-r/oth/0A/0E/R0A0E0000010001PDFE.pdf

ITU-R (2011). List of QUESTIONS assigned to ITU-R Study Group 5, http://www.itu.int/pub/R-QUE-SG05/en

Mitola, J. (20000), An Integrated Agent Architecture for Software Defined Radio, Dissertation Royal Institue of Technology, Sweden, ISSN 1403-5286, 8 May 2000.

Mody, A. And Chouinard, M. (2010). Enabling Rural Broadband Wireless Acess Using Cognitve Radio Technology, IEEE 802 Doc. 802.22-10/0073r03, available at: http:/ / www.ieee802.org/22/Technology/22-10-0073-03-0000-802-22-overviewand-core-technologies.pdf

Mueck, M. (2010a). ETSI Reconfigurable Radio Systems: Status and Future Directions on Software Defined Radio and Cognitive Radio Standards. IEEE Comm. Mag., September 2010.

Mueck, M. (2010b). TC RRS Activity Report 2010. http:/ / portal.etsi.org/rrs/activityreport2010.asp

NTIA (2011). Manual of Regulations and Procedures for Federal Radio Frequency Management (Redbook), US Government Printing Office Bookstore, Stock Number 903-008-00000-8 ISBN 0-16-016464-8

Prasad, V., Pawelczak, P., and Hoffmeyer, J., (2008). Cognitive Functionality in Next Generation Wireless Networks: Standardization Efforts, IEEE Comm. Mag., April 2008

Sherman, M., Mody, A., Martinez, R., Rodriguez, C., Reddy, R. (2008). IEEE Standards Supporting Cognitive Radioi and Dyanmic Spectrum Access, IEEE Comm. Mag., July 2008..

Stanislav, R., Harada, H., Murakami, H. And Ishizu, K. (2011), International Standardization of Cognitive Radio Systems, IEEE Comm. Mag., March 2011.

Stevenson, C. , Chouinard, G., Lei, Z,, Hu, W, Shellhammer, S., Caldwell, W., (2009). IEEE 802.22: The First Cognitve Radio Wireless Regional Area Network Standard, IEEE Comm. Mag., January 2009. http://www.inf.ufrgs.br/ cbboth/802_22commag.pdf 


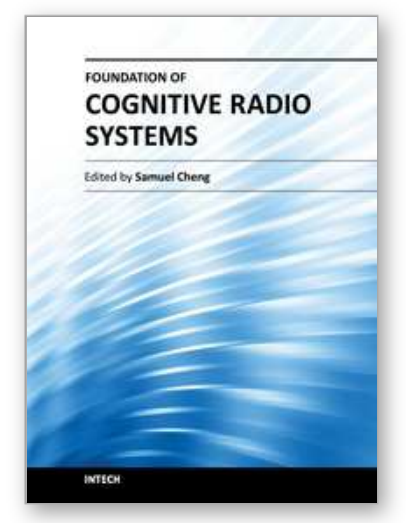

\author{
Foundation of Cognitive Radio Systems \\ Edited by Prof. Samuel Cheng
}

ISBN 978-953-51-0268-7

Hard cover, 298 pages

Publisher InTech

Published online 16, March, 2012

Published in print edition March, 2012

The fast user growth in wireless communications has created significant demands for new wireless services in both the licensed and unlicensed frequency spectra. Since many spectra are not fully utilized most of the time, cognitive radio, as a form of spectrum reuse, can be an effective means to significantly boost communications resources. Since its introduction in late last century, cognitive radio has attracted wide attention from academics to industry. Despite the efforts from the research community, there are still many issues of applying it in practice. This books is an attempt to cover some of the open issues across the area and introduce some insight to many of the problems. It contains thirteen chapters written by experts across the globe covering topics including spectrum sensing fundamental, cooperative sensing, spectrum management, and interaction among users.

\title{
How to reference
}

In order to correctly reference this scholarly work, feel free to copy and paste the following:

Jim Hoffmeyer (2012). A Roadmap to International Standards Development for Cognitive Radio Systems and Dynamic Spectrum Access, Foundation of Cognitive Radio Systems, Prof. Samuel Cheng (Ed.), ISBN: 978953-51-0268-7, InTech, Available from: http://www.intechopen.com/books/foundation-of-cognitive-radiosystems/international-standards-development-for-cognitive-radio-systems-and-dynamic-spectrum-access

\section{INTECH}

open science | open minds

\author{
InTech Europe \\ University Campus STeP Ri \\ Slavka Krautzeka 83/A \\ 51000 Rijeka, Croatia \\ Phone: +385 (51) 770447 \\ Fax: +385 (51) 686166 \\ www.intechopen.com
}

\author{
InTech China \\ Unit 405, Office Block, Hotel Equatorial Shanghai \\ No.65, Yan An Road (West), Shanghai, 200040, China \\ 中国上海市延安西路65号上海国际贵都大饭店办公楼405单元 \\ Phone: +86-21-62489820 \\ Fax: +86-21-62489821
}


(C) 2012 The Author(s). Licensee IntechOpen. This is an open access article distributed under the terms of the Creative Commons Attribution 3.0 License, which permits unrestricted use, distribution, and reproduction in any medium, provided the original work is properly cited. 